\title{
PERFORMANCE AND LONG TERM FORECAST MORBIDITI OF SENILE CATARACT IN THE AMUR REGION
}

Vydrov A.S., Komarovskikh E.N., Piskun V.E.

\section{Amur State Medical Academy, Blagoveshchensk, Russia}

Abstract. Among the world's population the number of elderly people is increasing progressively. In the period of 1980-2020 the expected increase in the elderly population in developed countries will be $186 \%$ in developing countries - $356 \%$. Experience of ophthalmic practice shows that the most common cause of blindness and visual impairment in older age groups is senile cataract. A retrospective analysis of data of annual statistical reports for LPU 1990-2013 is provided in the Amur medical information-analytical center. We analyzed the general and primary morbidity of senile cataract. Indicators calculated were based on the classical formulas per 1000 population per mille (\%o)Primary morbidity study in nosology has increased by $400 \%$ from $0,8 \%$ (1990) to $4 \%$ (2013). Throughout the period there was a dynamic growth rates of both general and primary morbidity of senile cataract. In an average year, the overall incidence was increased by $28.9 \%, 16.7 \%$ of primary-on.

Key words: cataract, the dynamics of the disease, prognosis

Introduction. Among the world's population the number of elderly people is increasing progressively. In the period 1980-2020 gg. the expected increase in the elderly population in developed countries will be $186 \%$ in developing countries - 356\%. Experience ophthalmic practice shows that the most common cause of blindness and visual impairment in older age groups is senile cataract. On this basis, WHO believes that in 2025 some 50 million people aged 60 will have varying degrees of intensity of lens opacity. [1] Of these, about 17 million are in need of surgical treatment[2].

Materials and methods. A retrospective analysis of data of annual statistical reports for LPU 1990-2013 gg. Provided in the Amur medical information-analytical center. We analyzed the general and primary morbidity of senile cataract. Indicators calculated based on the classical formulas per 1000 population per mille (\%o). In constructing the logarithmic trend line points by calculating the least-squares method using a logarithmic mathematical formula: $y=c \ln (x)+b$, where $a$ and $b$ - constant and In- natural logarithm function, and $X$ - time forecasting. In the simulation calculated the medium-term forecast for 5 years. results of the study/We've traced the dynamics of the general and primary morbidity of senile cataract population of the Amur region from 1990 to 2013. The overall incidence of cataracts residents of the Amur region during the analyzed period increased by $693.3 \%$ to $3,3 \%$ in 1990 to $26.2 \%$ in 2013 year. Primary morbidity study nosology has increased by $400 \%$ from $0,8 \%$ o (1990) to $4 \%$ o (2013).

Discussion of findings. Throughout the period there was a dynamic growth rates of both general and primary morbidity of senile cataract. In an average year, the overall incidence was increased by $28.9 \%, 16.7 \%$ of primary-on. Also, in the study of the dynamics of the general and primary morbidity of senile cataract population of the Amur region the trend line was obtained with the forecast of 5 years, which allows reliably identify $86-88 \%$ change of this index with a mathematical value: the overall incidence $=8,746 \ln (X)-4,231, R 2=0.858$; primary morbidity $=1,349 \ln (X)+0,303, R 2=0,878$. Where $X$ - the time period for which the forecast is required of disease; R2 - the degree of reliability of approximation. According to the forecast, to 2018 godu level of overall incidence of cataract may reach $39.5 \%$ with $86 \%$ certainty. As for the primary disease, its level in 2018 may reach $7 \%$ with a probability of $88 \%$ (Fig. 1) Preparation us incidence of senile cataract population of the Amur region as a whole in line with those in the country.

Conclusions. Thus, the problem of the incidence of senile cataract in the Amur region remains highly relevant, despite the high level of diagnostics and introduction of new technologies in the treatment of patients. Dynamics of general and primary morbidity of senile cataract population of the Amur Region is "aggressive" character. According to the medium-term forecast, the incidence of age-related cataract in the next 5 years in the Amur region will progressively increase, which requires the adoption of appropriate organizational and medical measures.

\section{Literature}

1. Aznabayev BM Ultrasonic phacoemulsification cataract. - M .: August Borg, 2005.136 p.

2. Libman ES, Shakhov EV Blindness and vision disability on the population of Russia // Proc. rep .: VIII Congress of Russian Ophthalmologists. M., 2005, pp 428-429

DOI:10.22448/AMJ.2016.15-16.120-122

UDC 613.955:616-053.5-071.3:612.143

FEATURES OF ACTIVITY OF CARDIOVASCULAR SYSTEM IN ADOLESCENT

Yutkina O.S., Babtseva A.F. 


\section{Amur State Medical Academy, Blagoveshchensk, Russia}

Abstracts. The study included 226 adolescents who were divided into 3 groups, depending on the somatotype of the child. The paper analyses AKDS card (automated complex dispensary surveys), calculated indices, characterizing the contractile activity of the heart, the work of the myocardium, describing the type of self-regulation of the circulatory system and others. The level of physical health is largely determined by the spare capacity of the cardiovascular system and differs significantly in adolescents with different somatotype.

Key words: children, cardiovascular system, somatotype.

Protection of children and young people's health is an important goal of medicine, The most universal indicator of the functional state of the organism and its compensatory and adaptive functions of the cardiorespiratory system is involved in the adaptation of children to environmental conditions and in the formation of the body's reaction to the impact of various factors $[1,2]$.

The formation of children's body, 10-14 years old, continues to further, characterized by a high rate of growth, weight gain, the intensity of metabolic processes. At this age, the forming of the skeleton and skeletal muscle as well as cardiovascular, pulmonary system, the digestive tract, the immunological defense system. A distinctive feature of this period of life is the restructuring of the neuro-endocrine sphere and, in particular, puberty [3].

For the first time, Soviet scientists Dorokhov and Bachrach offered somatotypes definition. According to Vorontsov, somatic growth means characteristic tempo: mikrosomatotip - slow, makrosomatotip - accelerated, mezosomatotip - the average growth rate. Based on his knowledge, you can accurately predict the rate of maturation and aging of the body, especially reactivity to a variety of external environment.

In adolescents the whole organism develops more active than in adults, however the total amount of blood in relation to body weight, they are more and larger than the value of shock and cardiac output with respect to a unit of body weight. However, the absolute magnitude of the stroke and minute blood volume in adolescents significantly less than in adults. The strength and the heart rate is greater in children than in adults. Thus, the pulse rate at rest have 88-90 beats per minute. In adolescents, still relatively wide openings in the heart and vascular lumen, and therefore facilitates the movement of blood, the conditions for greater speed and less flow resistance of peripheral vessels $[4,5,6]$.

Materials and methods. The purpose of this work - to determine the characteristics of the cardiovascular system in children 10-14 years old. 226 teenagers were examined, which were divided into 3 groups, depending on the somatotype of the child. The paper analyzes AKDS card (automated complex dispensary surveys), and calculated indices, characterizing the contractile activity of the heart, the work of the myocardium, describing the type of self-regulation of blood circulation, etc. Data indices are:. Systolic volume (Starr's formula), minute volume of blood, factor circulatory efficiency, the type of self-regulation of the circulatory, cardiac index (the index of the volume flow rate of the heart), specific peripheral vascular resistance, myocardial tension index (index Rufe), the external work of the myocardium, the efficiency criterion infarction left ventricular power index and others.

For example, the blood volume pumped by each ventricle into the main vessel (aorta or pulmonary artery) as a reduction of the heart, referred to as systolic (stroke) volume of blood. At rest, the volume of blood ejected from the ventricle is normally a third to a half of the total amount of blood contained in the chamber of the heart at the end of diastole. Remaining in the heart of the reserve after the systole blood volume is the original depot, providing an increase in cardiac output during situations that require rapid intensification of hemodynamics (for example, during physical exertion, emotional stress, etc.). The value of the reserve volume of blood is one of the main determinants of the functional reserve of the heart on its specific function - the movement of blood in the system. By increasing the reserve volume accordingly increases systolic maximum volume that may be ejected from the heart under conditions of intense activity. When adaptive reactions circulatory apparatus changes in systolic volume are achieved through self-regulation mechanisms under the influence of extracardiac neural mechanisms. Regulatory effect realized in systolic volume change by acting on the contractile force of the myocardium.

Results and discussion. All the children were divided into 3 groups: Group 1 - 71 children with low physical development ( 43 girls and 28 boys), Group 2 - with an average physical development - 67 children (35 girls and 32 boys), Group 3 - 68 children with high physical development ( 42 girls and 26 boys).

In the study group of teenagers 10-14 years old, mezosomatotype children have lower values of heart rate $(81,72 \pm 0,8$ beats / min.), As well as lower levels of systolic and diastolic blood pressure (SBP $=97,39 \pm 08$ $\mathrm{mmHg}, \mathrm{DBP}=63.28 \pm 0.9 \mathrm{~mm} \mathrm{Hg})(\mathrm{p} \square 0,05)$. macrosomatotype children have observed significantly higher values as the heart rate $(84,62 \pm 0,6$ beats / $\mathrm{min})$ and $\operatorname{SAD}(103,6 \pm 1,2 \mathrm{~mm} \mathrm{Hg})$, diastolic blood pressure $(68,38$ $\pm 0,7 \mathrm{~mm} \mathrm{Hg})(\mathrm{p} \square \mathrm{o}, 05)$. It means, that macrosomatotype children's cardiac muscle work in the least-saving mode and the range of compensatory possibilities of the organism is limited.

The indicators characterizing the contractile activity of the heart, in addition to heart rate, cardiac output is the value of the blood flow and the value of the systolic volume. Minute volume of blood (IOC) - this is the 
amount of blood ejected by the ventricles per 1 minute (rate - 3.5-5.5 l). Systolic volume of the heart (CO) - is the amount of blood ejected by the heart ventricles at the same reduction (norm - 50-90 ml). Children with mezosomatotype physique revealed the highest IOC indicators and CO $(5,43 \pm 0,4 \mathrm{l}$ and $66,31 \pm 0,3 \mathrm{ml}$, respectively) $(\mathrm{p} \square \mathrm{o}, 05)$. This indicates a high power of heart contractions. The lowest rates were found in macrosomatotype groups $(\mathrm{IOC}=5,38 \pm 0,2 \mathrm{l}, \mathrm{CO}=63,98 \pm 0,6 \mathrm{ml})$ and microsomatotype groups $(\mathrm{IOC}=5,11 \pm 0,4 \mathrm{l}, \mathrm{CO}=62,61 \pm 1,0$ $\mathrm{ml})$, but the values are within the norm (p円o,05).

Current index adaptive capacity of the cardiovascular system by R.M.Baevskomu (AP) defines the functional status of the patient. Children of all somatotypes index below 2.6, indicating that a satisfactory adaptation of teenagers, high reserve capacity of the heart.

Coefficient efficiency circulation (KEK) is defined as: KEK = (SBP, DBP), heart rate. It describes the costs of the organism in the blood travel in the bloodstream. It was found that macrosomatotype children economical happens spending reserves of the cardiovascular system (indicator KEK $=2975,4 \pm 0,8$ ), compared with microsomatotype children $(K E K=2706,5 \pm 1,0)$ and mezosomatotype children $(K E K=2794 \cdot 5 \pm 1,2)(p \square 0,05)$.

Rufe index reflects the adaptive capacity of the cardiovascular system in response to a graduated exercise. Children of all somatotypes Rufe index showed a below-average level of reserve of the cardiovascular system. Significantly better results were observed in students with mezosomatotype physique.

Determining the type of self-circulation provides an opportunity to assess the level of stress in the regulation of the cardiovascular system. In adolescents, all somatotypes TSC index less than 90 (micro - 84,33 \pm 0,5 ; mezo $-78,82 \pm 0,8$; macro $-82,47 \pm 0,4$ ), which indicates the type of self-regulation of cardiac blood flow (pD 0.05).

Conclusions. Thus, the level of physical health is largely determined by the spare capacity of the cardiovascular system and differ significantly in adolescents with different somatotypes (slow, normal and accelerated). Children with microsomatotype and microsomatotype body type, can be attributed to the group of persons with reduced functional reserves of the cardiorespiratory system and lower compensatory capacity of the organism in response to exercise. Establishing a relationship of morphological and functional characteristics of children of different types of constitution, allows the further development of a fundamental framework for a more complete study of the physiological individuality and proposals for the development of new approaches to preclinical diagnosis of disorders of the cardiorespiratory system and evaluation of the general state of their health.

\section{Literature}

1. Yutkina O.S. The functional state of cardio-respiratory system in children, depending on the somatotype. In: Proceedings of the VI Congress of doctors pulmonologists Siberia and the Far East. Far Eastern Scientific Center of Physiology and Pathology of respiration, Amur State Medical Academy, Ministry of Health of the Amur region, Russian respiratory society. 2015. pp 157-161.

2. Yutkina O.S. Status of the cardiorespiratory system in adolescents depending on somatotype. In: Evidence-based medicine - the basis of modern health care. Collection of scientific papers (in the framework of the National Year of combating cardiovascular disease). Institute of Advanced Training of health professionals. 2015. pp 41-43.

3. Yutkina O.S. Constitutional features of children, depending on the physical development.// International scientific review. Number 2015. 2 (3). S. 84-86.

4. Yutkina O.S., Babtsev A.F., Arutyunyan K. The health of children and adolescents based on the results of the automated complex dispensary examination.// Allergology and Immunology. 2014. Vol 15. №2. P.119.

5. Yutkina O.S. Circadian blood pressure profile in younger schoolboys // Proceedings of the V International Scientific and Practical Conference "21st Century: basic science and technology." 2014. Volume 1. pp 45-47.

6. Yutkina O.S., Babtsev A.F., T.A. Metelkina, Amelichkina T.V., Murrieva N.V. Early markers of hemodynamic disorders in children, depending on the physical development of the Amur // Medical Journal. 2014. number 4 (8). S. 84-86.

DOI:10.22448/AMJ.2016.15-16.122-124

UDC 616-053.5:612.112.94

\section{ADAPTATION REACTIONS OF JUNIOR SCHOOL CHILDREN}

Yutkina 0.S.

\section{Amur State Medical Academy, Blagoveshchensk, Russia}

Abstract Non-specific types of adaptive reactions of an organism of children of 6-9 years old were estimated. The analysis of maps of hardware and software inspection of dispensary children was maden. The total number of study included 192 people. The adaptive response of the body in children, depending on the age and gender, was studied using data leukochogram (percentage of lymphocytes in the leucocyte count) (Garkavi, 1998). 